The BMJ

emahase@bmj.com

Cite this as: $B M J 2020 ; 371: \mathrm{m} 4042$ http://dx.doi.org/10.1136/bmj.m4042 Published: 22 October 2020

\section{Covid-19: Vaccine trials need more transparency to enable scrutiny and earn public trust, say experts}

\begin{abstract}
Eleven covid-19 vaccine candidates are in phase III clinical trials. But while the scientists involved have been praised for their speed, others are calling for more transparency to ensure studies are robust and the evidence is sound. Elisabeth Mahase reports
\end{abstract}

\section{Elisabeth Mahase clinical reporter}

“These are unprecedented times and these are unprecedented clinical trials ... This can't be business as usual," says Jason Schwartz, assistant professor of public health at Yale School of Public Health.

Schwartz says that transparency is crucial to ensure that the scientific community can review and scrutinise the work but also to build public understanding and confidence in the much anticipated potential covid-19 vaccines.

However, not all transparency is equal, he adds. "It needs to be the kind of transparency that isn't filtered through corporate press releases or statements to investors, or a kind of pseudo-transparency in which the manufacturers are trying to massage or control the message. [It needs to be] real transparency in terms of the kinds of documents and materials that can help the public, and especially the broader scientific community, both understand the design of these trials and ultimately understand how to interpret the results from them."

\section{Show your working}

A key document to understanding how trials are being run is the study protocol. After pressure from scientists and public health experts, four major protocols of covid vaccine trials have now been made public. These include AstraZeneca's halted US study of the University of Oxford's AZD1222 vaccine candidate, ${ }^{1}$ the Pfizer and BioNTech study of their BNT162b, ${ }^{2}$ Moderna's study of its mRNA-1273, ${ }^{3}$ and the Johnson \& Johnson Ad26.COV2.S. ${ }^{4}$

Additionally, the Oxford team has published the information sheets provided to trial participants, ${ }^{5} \mathrm{a}$ move towards transparency that other companies seem not to have followed. Moderna, Pfizer, and BioNTech did not respond to several $B M J$ requests for the informed consent forms or offer an explanation as to why they would not publish them.

Schwartz credits those trial sponsors that have listened. "The release of the protocols was really important and, as far as I know, an unprecedented step so early in the conduct of the trial ... The consent documents are certainly important to help us understand the ethics of these trials and help understand how the research subjects are being informed about their participation and the risks and benefits of it."

However, he added that there was plenty more that could be shared in the form of real objective documents about plans, relationships, and scientific standards. Schwartz has called for trial sponsors to share these raw materials to "help the scientific community understand the work that's ongoing and help the public understand what is usually a process that rarely gets this kind of widespread scrutiny."

\section{Who has oversight?}

One key area that Schwartz is interested in relates to the independent boards that review the data from these clinical trials. "These data and safety monitoring boards [DSMBs] will be undertaking those interim reviews of the trials and potentially stopping the trial because the evidence is so favourable or, conversely, the evidence is so unfavourable or there's a serious safety issue. DSMBs are so important, particularly if these vaccines get an expedited review and potential authorisation here in the US by the Food and Drug Administration,” he explains.

Despite the importance of the DSMBs, little is known about their standards. "There's more we can learn about the groups and their approaches [and] plans, since that seems to be a really important lever," says Schwartz.

In the US a joint DSMB has been formed to review unblinded data from several trials being run in the country, including the Moderna, Johnson \& Johnson, and AstraZeneca trials, but not Pfizer's. Although the identity of the board's 10-15 members has mostly remained confidential, the chair-appointed by the director of the National Institute of Allergy and Infectious Diseases, Anthony Fauci-has been revealed as Richard Whitley. Whitley is a professor of paediatrics and co-division director of paediatric infectious diseases at the University of Alabama. He also sits on the board of directors of the drug company Gilead, for which he was paid roughly $\$ 430$ 000 in 2019. 6

The bioethicist Charles Weijer, who specialises in the ethics of health related randomised controlled trials at Western University, Ontario, thinks the entire membership of these DSMBs should be made public. "Public confidence in judgments [made by these groups] will be enhanced by knowing that committee members possess requisite expertise and have no relevant conflicts of interest. While this is not usual practice in clinical trials, this step is required to bolster public trust in covid-19 vaccine research." Weijer receives consulting income from the drug company Eli Lilly. 
Schwartz would also like to see more information made public on the contractual agreements between manufacturers and their government sponsors. "[These agreements] have been shielded from public view, and I think that's detrimental to understanding the nature of these relationships and partnerships," he says.

\section{Ending trials early}

A key role that DSMBs could play is in stopping vaccine trials early. Fauci, who advises the White House on covid-19, has said this can happen if the boards decide the data are good and a vaccine is safe and effective. He emphasised that those making such a decision "better be sure" that the evidence was good but said there would be a "moral obligation" to get the vaccine to the public earlier if it really was safe and effective. ${ }^{7}$

At this point, Schwartz says transparency will be crucial. "The real critical moment and opportunity for transparency will be when we get to the point where decisions by regulatory bodies are going to be made based on the products in these trials. And I think that's where the light will and should shine, with even greater focus."

Being transparent about how such a decision is made will be even more important given the unease that has built up that a vaccine might be approved prematurely. Concerns have grown over potential political pressure to rush out a vaccine before the science is clear since comments made by Donald Trump, in which he hinted that a vaccine could be made available before the 3 November presidential election. ${ }^{8}$

This seemed to have prompted the heads of nine drug companies-AstraZeneca, BioNTech, GlaxoSmithKline, Johnson \& Johnson, Merck/MSD, Moderna, Novavax, Pfizer, and Sanofi-to sign an open letter committing to submit for approval or emergency use authorisation only after "demonstrating safety and efficacy through a Phase 3 clinical study that is designed and conducted to meet requirements of expert regulatory authorities such as FDA [the

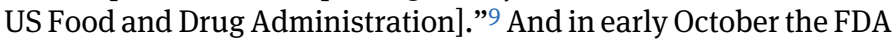
published new guidance stating that it would require at least two months of follow-up data after trial participants received all doses of a vaccine, leading many people to conclude that a so called "October surprise" was no longer possible. ${ }^{10}$

But Weijer thinks that those responsible should go further and publicly confirm that they will not end trials early if they get a positive efficacy signal during interim analysis.

"We really need safety information on the full planned sample size in order to give us the sort of good grounds to believe not only that a vaccine works but, crucially, that it's safe," he says. "We're going to be giving this vaccine to hundreds of millions of healthy people. To be able to reliably detect a very rare adverse event which is associated with the vaccine-so that's something that occurs in one in 10 ooo people-you need safety data on 60 ooo people," he says.

The importance of ensuring that vaccines are not rushed out is something emphasised by experts from the UK Royal Society's DELVE (Data Evaluation and Learning for Viral Epidemics) initiative, ${ }^{11}$ which has published a report on the development and implementation of covid-19 vaccines. ${ }^{12}$ "If vaccines were deployed outside clinical trials before safety and efficacy have been fully established and prove to be ineffective or cause rare but severe side effects during the larger-scale roll-out, they could cause substantial harm and damage public confidence in other vaccines," DELVE warned.

The DELVE team also highlighted the importance of transparency when it comes to the limitations of any vaccine: "Clear, transparent communication can be used to address rational doubts and to enable informed decision-making ... it should not hide the potential limitations of vaccines including possible limited availability, incomplete protection requiring boosting, and reactogenicity."

The report says that although "negative or complicating factors might lower uptake, their discovery post-rollout is likely to have a far greater negative impact on uptake.”

\section{Trial transparency gone awry}

In September the University of Oxford and AstraZeneca's vaccine candidate made headlines after the trials were paused around the world (including in the US, UK, Japan, India, Brazil, and South Africa) while an investigation was carried out into a "single event of an unexplained illness." Experts have made it clear that pauses in vaccine trials are common and a sign that the trial's oversight process is working. ${ }^{13}$

While this was the first time a pause of the trial had been announced, it was not actually the first time it had been halted. The patient information sheet from 12 July shows that it was paused earlier in the year after one trial volunteer developed symptoms of transverse myelitis. ${ }^{14}$

But bioethicist Charles Weijer of Western University, Ontario, told The $B M J$ that the disclosure of the serious adverse events in the Oxford trial patient information sheet "is not, in my view, adequate."

When he compared different versions of the sheet, ${ }^{15}$ he noted that while transverse myelitis was initially mentioned, this was then removed and replaced with "vaguer language." The 11 September version said that volunteers had "developed unexplained neurological symptoms including changed sensation or limb weakness." It was reported in the media that the second serious adverse event in September may have been transverse myelitis, although this was not confirmed, and that the patient was admitted to hospital as a result. It was then reported that the patient was set to be discharged from hospital, but no further updates have been provided.

"Prospective participants are not informed that, if media reports are to be believed, the second case of transverse myelitis was sufficiently severe as to warrant hospital admission," says Weijer. "Consent documents should contain a clear and accurate description of any serious adverse events. Certainly, they should contain more information than a prospective or current participant could discover with a Google search of the vaccine trial."

The handling of the trial pause has also been criticised in terms of how the information was communicated to the public. "It's transparency gone awry," says Jason Schwartz, assistant professor of public health at Yale School of Public Health. "We know we've received only very, very fragmentary information about the nature of the events that occurred. Details about what it was were mostly, as I understand it, made public through a call to investors ... I think there was a failure in terms of how there were just drips of information and uncertainty and missing details to help contextualise what that signal was."

Trials of this vaccine have resumed in Japan, the UK, Brazil, South Africa, and India. Despite this, AstraZeneca's US based trial has not been restarted, with the Food and Drug Administration recently broadening its safety investigation. ${ }^{16}$

But this is not the only trial paused because of adverse events. On 12 October Johnson \& Johnson announced that it had paused dosing in all its covid-19 vaccine trials while an investigation was carried out into an unexplained illness in a study participant. The company did not seem to be upfront about the issue: the pause was first revealed by the US media outlet STAT. ${ }^{17}$ It remains to be seen whether lessons from the Oxford and AstraZeneca trial have been learnt.

Competing interests: None declared.

Not externally peer reviewed. 
1 AstraZeneca. A phase III randomized, double-blind, placebo-controlled multicenter study in adults to determine the safety, efficacy, and immunogenicity of AZD1222, a non-replicating ChAdOx1 vector vaccine, for the prevention of COVID-19. https://s3.amazonaws.com/ctr-med-

7111/D8110C00001/52bec400-80f6-4c1b-8791-0483923d0867/c8070a4e-6a9d-46f9-8c32cece903592b9/D8110C00001_CSP-v2.pdf.

2 Pfizer. A phase $1 / 2 / 3$, placebo-controlled, randomized, observer-blind, dose-finding study to evaluate the safety, tolerability, immunogenicity, and efficacy of SARS-CoV-2 RNA vaccine candidates against covid-19 in healthy individuals. https://pfe-pfizercom-d8-prod.s3.amazonaws.com/2020-09/C4591001_Clinical_Protocol.pdf.

3 Moderna. A phase 3, randomized, stratified, observer-blind, placebo-controlled study to evaluate the efficacy, safety, and immunogenicity of mRNA-1273 SARS-CoV-2 vaccine in adults aged 18 years and older. https://www.modernatx.com/sites/default/files/mRNA-1273-P301-Protocol.pdf.

4 Janssen Vaccines \& Prevention BV. A randomized, double-blind, placebo-controlled phase 3 study to assess the efficacy and safety of Ad26.COV2.S for the prevention of

SARS-CoV-2-mediated COVID-19 in adults aged 18 years and older. https://www.jnj.com/coronavirus/covid-19-phase-3-study-clinical-protocol.

5 University of Oxford. COVID-19 vaccine study (COV002). COVID-19 Vaccine Study (COV002). https://www.covid19vaccinetrial.co.uk/participate-oxford.

6 Pradhan R. These secret safety panels will pick the covid vaccine winners. KHN. 24 Sep 2020. https://khn.org/news/these-secret-safety-panels-will-pick-the-covid-vaccine-winners

7 Szabo L. Fauci says COVID vaccine trials could end early if results are overwhelming. 1Sep 2020 https://khn.org/news/dr-fauci-says-covid-vaccine-trials-could-end-early-if-results-are-overwhelming.

8 Trump says a coronavirus vaccine could be ready in October at a White House briefing. YouTube 7 Sep 2020. https://www.youtube.com/watch?v=YDDvNsEupbw\&feature=youtu.be.

9 Dyer O. Covid-19: Pharma companies promise not to bow to political pressure to rush vaccine production. BMJ2020;370:m3512. doi: 10.1136/bmj.m3512 pmid: 32909956

10 Food and Drug Administration. Emergency use authorization for vaccines to prevent covid-19: guidance for industry. Oct 2020. https://www.fda.gov/media/142749/download.

11 Royal Society DELVE Initiative. People. https://rs-delve.github.io/people.html.

12 Royal Society DELVE Initiative. Testing and evaluation of vaccine candidates. https://rsdelve.github.io/reports/2020/10/01/covid19-vaccination-report.html\#testing-and-evaluation-ofvaccine-candidates.

13 Mahase E. Covid-19: Oxford researchers halt vaccine trial while adverse reaction is investigated. BMJ2020;370:m3525. doi: 10.1136/bmj.m3525 pmid: 32907856

14 University Hospitals Birmingham NHS Foundation Trust. Participant information sheet: COV002. https://www.covid19vaccinetrial.co.uk/files/cov002pisages18-55yearsv8012jul20-birmpdf.

15 ISRCTN Registry. Investigating a vaccine against COVID-19. http://www. isrctn.com/ISRCTN90906759.

16 Taylor M, Levine D. Exclusive: FDA widens US safety inquiry into AstraZeneca coronavirus vaccine-sources. Reuters. 30 Sep 2020. https://uk.reuters.com/article/health-coronavirus-vaccineastrazeneca/exclusive-fda-widens-us-safety-inquiry-into-astrazeneca-coronavirus-vaccinesources-idUKL1N2GR1SK.

17 Herper M. Johnson \& Johnson Covid-19 vaccine study paused due to unexplained illness in participant. Stat 2020. https://www.statnews.com/2020/10/12/johnson-johnson-covid-19-vaccinestudy-paused-due-to-unexplained-illness-in-participant.

This article is made freely available for use in accordance with BMJ's website terms and conditions for the duration of the covid-19 pandemic or until otherwise determined by BMJ. You may use, download and print the article for any lawful, non-commercial purpose (including text and data mining) provided that all copyright notices and trade marks are retained. 